\title{
L'orientation dans les techniques du corps chez les Mongols
}

Orientation refering to the techniques of the body

Gaëlle Lacaze

\section{OpenEdition}

1 Journals

\section{Édition électronique}

URL : https://journals.openedition.org/emscat/810

DOI : $10.4000 /$ emscat. 810

ISSN : 2101-0013

\section{Éditeur}

Centre d'Etudes Mongoles \& Sibériennes / École Pratique des Hautes Études

\section{Édition imprimée}

Date de publication : 1 mai 2006

Pagination : 163-205

ISBN : 2-9518888-3-X

ISSN : 0766-5075

\section{Référence électronique}

Gaëlle Lacaze, «L'orientation dans les techniques du corps chez les Mongols », Études mongoles et sibériennes, centrasiatiques et tibétaines [En ligne], 36-37 |2006, mis en ligne le 17 mars 2009, consulté le 13 juillet 2021. URL : http://journals.openedition.org/emscat/810 ; DOI : https://doi.org/10.4000/ emscat.810

Ce document a été généré automatiquement le 13 juillet 2021.

(c) Tous droits réservés 


\title{
L'orientation dans les techniques du corps chez les Mongols
}

Orientation refering to the techniques of the body

\author{
Gaëlle Lacaze
}

\section{NOTE DE L'AUTEUR}

Je remercie chaleureusement R. Hamayon et $F$. Aubin pour leurs relectures de cet article et les critiques qu'elles en ont formulées. Cet article est basé sur l'analyse des conceptions de l'espace centrées sur le corps, développée dans ma thèse de doctorat (2000) sur les « Techniques et les représentations du corps chez des peuples mongols ", dirigée par R. Hamayon, et approfondie depuis dans d'autres recherches. [Note du rédacteur en chef : le présent article est publié dans EMSCAT 36-37, sous la responsabilité scientifique de $\mathrm{M}^{\text {mes }} \mathrm{F}$. Aubin et R. Hamayon.]

1 Parmi les «techniques du corps " ${ }^{1}$, celles de « l'activité, du mouvement » occupent une place centrale. Cependant, hormis les techniques sportives et chorégraphiques, elles ne retiennent généralement pas l'attention des observateurs. Les travaux consacrés aux manières de marcher ${ }^{2}$ et de s'arrêter ou aux manières de s'asseoir ${ }^{3}$ et de dormir sont rares. À l'exception des techniques de mouvement associées aux activités économiques (par exemple, la traite, la tonte, le gardiennage, etc.), ces techniques ont été peu étudiées chez les populations d'Asie centrale et septentrionale, turcophones ou mongolophones, et les sources écrites sur l'ensemble des techniques du corps liées à l'activité et au mouvement sont inexistantes. Le silence pesant sur leur aspect ethnographique pourrait provenir du fait qu'elles sont, de prime abord, ressenties et considérées comme naturelles par les autochtones et les observateurs étrangers. Elles sont pourtant pertinentes pour une étude de l'orientation.

Déjà des travaux de linguistique mongole ont mis en évidence l'intérêt que présentent les notions de mobilité et d'orientation pour comprendre les sociétés de pasteurs nomades ${ }^{4}$. Dans cet article, nous proposons d'étudier les règles gouvernant les 
techniques de veille et de mouvement chez les Mongols. Très précises, ces règles organisent la gestuelle en fonction du sexe, de l'âge et du statut, attribuant à chaque catégorie de personne une gestuelle propre pour chaque technique du corps. Elles révèlent des représentations de l'espace centrées sur le corps, ou, selon les termes de C. Humphrey, « ego-centrées» (1995, p. 142). Cela ne signifie pas que l'espace est pensé en fonction de catégories faisant nécessairement référence au corps humain, mais que la perception de l'espace par les "sens" du corps, selon la position du locuteur et ses déplacements, joue un rôle fondamental dans le système d'orientation. Ainsi, la perception de l'espace possèderait un caractère dynamique lié à la mobilité et à la vitalité du corps, le corps étant considéré comme support de force vitale. Dans cette perspective, les représentations spatiales fournies par les facteurs sensoriels, en fonction de la position du corps et de ses trajets possibles (par le déplacement, mais aussi par l'audition ou la vision) seraient dynamiques et prédomineraient sur les principes statiques d'orientation absolue ${ }^{5}$.

Nous illustrerons cette hypothèse en analysant d'abord les techniques de sommeil et de veille au repos (l'allongement, l'assise) et, ensuite, les techniques de mouvement (marche, saut, enjambement, monte). La distinction entre repos et mouvement recoupe, dans ses grandes lignes, la distinction entre la yourte et le plein air. Des techniques du corps spécifiques marquent le passage d'un lieu à un autre. Les conceptions du paysage seraient donc partiellement déterminées par la situation spatiale du corps durant ses parcours. Le paysage des éleveurs serait alors moins conçu par référence statique à des points d'orientation absolue qu'en termes dynamiques de mouvement et d'activité.

\section{Les techniques de repos et de veille: «Sous la yourte »}

4 En préalable, il est nécessaire de rappeler les principes de l'orientation chez les Mongols. Ceux du centre (en Mongolie xalx) s'orientent en regardant vers le Sud, la "droite ", baruun en cyrillique et baraүun en écriture classique, servant alors à désigner "l'ouest »; et la "gauche », züün en cyrillique et zegün en écriture classique, «l'est ». Mais, pour les Ordos de Chine qui orientent leur yourte en regardant vers l'est, baruun/ baraүun signifie le «sud» (Mostaert [1941-1944] 1965, p. 56) et zü̈̈n/zegün, le «nord» (ibid., p. 225). C'est l'usage qui attribue à baruun/züün, désignant la droite et la gauche, le sens d'ouest et d'est en Mongolie centrale. Le lien entre les termes d'orientation relative au corps et ceux désignant les points cardinaux est donc plus lié aux usages qu'issu d'une relation sémantique. Le rapport entre l'orientation relative et l'orientation absolue serait ainsi, de prime abord, lié à l'orientation du corps et à celle de la yourte dans l'espace. Il faut donc traduire baruun par «droite» et zü̈̈n, par " gauche».

\section{La droite et la gauche}

Chez les Mongols, les termes "droite " et "gauche " servent à exprimer des valeurs culturelles, morales et sociales. La droite est le côté ou la direction juste, le côté de l'aîné et de l'homme. La gauche renvoie, en revanche, à la femme et au cadet. Ce terme 
sert également à exprimer un geste maladroit ou une action erronée, l'infraction et la transgression (Beffa et Hamayon 1983, pp. 96-99).

Sur le terrain, force est de constater que tout échange social est impérativement effectué avec la main droite. La seule activité qui impose l'emploi de la main gauche est l'aération du lait par les femmes lors de sa première ébullition, tout de suite après la traite. L'emploi de la main gauche est alors préconisé «pour ne pas perdre la force vitale du foyer ». L'explication de cet usage par mes informateurs darxad ${ }^{6}$ fait systématiquement référence à l'orientation de la yourte : il faut orienter la coulée de lait dans la direction opposée à la porte, vers le fond, le xojmor (qojimar en écriture classique). Cet usage préférentiel de la main gauche ${ }^{7}$ 'implique aucun échange ; il s'oppose donc aux gestes liés à une forme de sociabilité qui sont, quant à eux, toujours effectués avec la main droite. Il renvoie à une volonté de préserver en interne la force vitale du groupe. La transmission «entre soi »/«à autrui » pourrait ainsi trouver des implications dans la latéralisation gauche/droite du corps.

\section{De la latéralisation du corps à l'orientation dans la yourte}

7 L'aménagement et l'organisation à l'intérieur de la yourte utilisent des termes d'orientation. On se place « au nord» ou vers le «sud» de la yourte; on s'installe du " côté gauche/est» ou "droit/ouest», etc. L'emploi de ces termes d'orientation est constant dans la conversation. Il tient compte à la fois de repères d'orientation absolue et de ceux fournis par les positions relatives des corps. Les parties «droite» et " gauche » de la yourte sont déterminées en fonction de la position idéale d'un locuteur tourné face à la porte et envisagé a priori en tant qu' « ancien ", c'est-à-dire placé dans le fond, dans la partie la plus estimée de la yourte, le xojmor. C'est par référence à cette position idéale que sont le plus souvent expliqués l'organisation interne et l'aménagement de la yourte.

8 En général, les membres de la famille s'installent du côté gauche, et les invités, du côté droit. Ces places sous la yourte sont en fait déterminées par l'orientation du poêle : la famille s'installe du côté de l'ouverture du poêle, sa «bouche » généralement tournée vers la gauche, et les invités, derrière le poêle, à droite. Certaines circonstances poussent à tourner le poêle de l'autre côté, la bouche se trouvant alors orientée vers la droite de la yourte. Ces « yourtes inversées » sont rares : elles sont aménagées en cas de décès du chef de famille ou quand une femme exerce la position dominante dans le foyer, par exemple chez certaines femmes chamanes ou dans certains lignages.

On ne s'assied jamais directement face à la bouche du poêle ou devant la porte, mais toujours avec un décalage sur le côté. Traditionnellement, le fond de la yourte, le xojmor, était occupé par un autel, donc derrière le poêle et à l'opposé de la porte. À l'époque communiste,on y posait les photographies d'anciens de la famille, morts ou vivants. Aujourd'hui, on y trouve en outre des représentations de divinités ou des livres bouddhiques. Et quand il n'y a pas d'autel proprement dit, on expose dans le xojmor des objets estimés, pour des raisons sentimentales, économiques ou sociales.

10 «L'autel » est posé sur un petit coffre en bois contenant des objets moins honorifiques mais néanmoins estimés, comme les petits bols ouvragés, les vêtements ou les instruments associés à des festivités. Selon les familles, il peut occuper le fond de la yourte ou seulement une partie de celui-ci. Il est alors plutôt placé à l'extrémité «droite» du fond de la yourte. On évite de lui tourner le dos, et donc de s'asseoir 
exactement devant lui, ce qui peut toutefois être le fait de notables, ou d'orienter ses pieds dans sa direction. L'orientation du corps sous la yourte correspond donc à celle de la yourte. On s'arrange pour avoir toujours le haut du corps du côté du fond de la yourte, du xojmor, et les pieds du côté de la porte. Aussi les manières de s'asseoir varient-elles pour respecter cette orientation parallèle du corps et de la yourte.

Quant à la position de la yourte dans l'espace, elle doit respecter un certain nombre de règles relatives à l'orientation de la porte et du xojmor. La porte doit faire face à la rivière quand il y en a une, être placée vers l'aval sur un flanc de montagne, dans la direction opposée au vent dans un défilé, ou en direction du sud dans une plaine. Autrement dit et quand cela est possible, le xojmor est placé en direction du nord ; mais quand la configuration du paysage l'impose, c'est-à-dire assez souvent hors du Gobi et des larges vallées de steppe, il est toujours orienté en direction des montagnes, dans la direction opposée à la rivière ou vers l'amont dans un défilé. L'orientation réelle de la yourte dépend donc de nombreux facteurs, même si elle est idéalement et linguistiquement orientée selon un axe nord/sud.

\section{L'allongement}

12 Le repos proprement dit est lié à l'allongement et au coucher, réservé à la périphérie intérieure de la yourte où se trouvent placés les lits. La position assise est adoptée au centre de la yourte où se trouvent la table et le poêle. Chacune de ces techniques du corps correspond donc à un lieu spécifique, qui lui est réservé. Ainsi, même si cela est concrètement possible, on ne peut ni manger au lit ni s'avachir ou s'endormir sur un tabouret ou une chaise. Chaque espace sous la yourte est marqué par une gestuelle propre et, en outre, nous le verrons, le passage d'un espace à l'autre suppose le franchissement d'une frontière symbolique qui appelle la pratique d'une technique du corps particulière.

13 Les lits sont placés au pourtour de la yourte. Il y a généralement un lit sur chacun des deux côtés. Parfois, un autre lit est installé dans le xojmor. De fins matelas en feutre peuvent être dépliés sur le sol la nuit venue, si le nombre de lits ne suffit pas. L'orientation des lits latéraux ou des matelas, et par conséquent du corps, suit autant que possible l'axe vertical de la yourte. La tête ${ }^{8}$ est placée vers le xojmor et les pieds, en direction de la porte, comme nous venons de le voir.

Le sommeil est un moment particulier, pour autant que l'absence de mouvement peut évoquer la mort. Il se caractérise par une relative absence de norme et par une faible spécialisation sexuelle et générationnelle. La position de sommeil privilégiée de l'adulte est l'allongement latéral. Le corps est alors tourné en direction des parois de la yourte, le dos faisant face au centre. Il y a ainsi opposition entre le centre de la yourte, lieu d'expression de la sociabilité, et le pourtour, espacé privé, permettant, grâce aux lits qui y sont disposés, la pratique des techniques de repos.

\section{S'étirer en se levant}

Autant la sphère privée du lit en bordure de la yourte laisse place à l'expression individuelle, autant la sphère publique de la table et du poêle en son centre est le lieu de normes précises et explicites. Ces deux sphères sont séparées par une frontière symbolique, correspondant à la distinction entre deux techniques du corps: 
l'allongement et l'assise. Mais entre les deux, une autre technique prend place. Au réveil, en effet, le passage de l'allongement à la position debout donne lieu à un geste particulier : l'étirement du corps en levant les bras. Après avoir dormi, ce geste est censé permettre la ré-incorporation de l'âme par les aisselles - l'âme étant censée s'évader du corps pendant le sommeil (ce que fonde l'expérience du rêve). L'étirement est pratiqué dans l'espace situé entre le lit et la table, le dos face au poêle. Tournée vers le lit, la personne qui s'étire n'est pas encore orientée vers l'espace public de la yourte, son centre. Accepté au sortir du sommeil, l'étirement est fortement prohibé en d'autres circonstances, car il pourrait entraîner le départ de l'âme. C'est donc une technique du corps marquant le passage entre les sphères privée et publique.

\section{S'asseoir}

16 Être sous la yourte en état de veille implique d'être assis, car il n'est pas convenable d'y rester debout. Et s'asseoir ouvre généralement sur la consommation alimentaire. Ainsi, le seul fait d'entrer dans une yourte impose pratiquement au visiteur de s'asseoir et de partager le thé ou le repas qu'on lui offre. L'assise et l'alimentation s'accompagnent en outre d'échanges de paroles. Assise, alimentation et communication sont donc liées entre elles et à la sociabilité.

Chacun prend position de chaque côté du poêle en descendant du xojmor vers la porte. Quelle que soit la place de chacun, le corps fait face au centre de la yourte et le dos est tourné en direction des murs. Plusieurs règles régissent les manières de s'asseoir pour respecter cette orientation selon la place où l'on s'installe. Les personnes assises dans le xojmor peuvent étendre leurs jambes en direction de la porte, en les décalant légèrement du côté des murs pour ne pas les diriger vers le poêle. Elles s'adossent aux meubles qui sont installés dans le fond de la yourte, mais évitent de le faire sur celui où l'autel est posé. Les personnes assises aux alentours du poêle lui font face et orientent leur corps parallèlement à l'axe vertical de la yourte. Ce sont généralement des cadets classificatoires, des femmes ou des jeunes gens, qui s'assoient en repliant leurs jambes sous les fesses. Pour étendre les jambes, il faut les diriger vers la porte, en les décalant vers le mur. Dans cette position, le corps est orienté dans deux directions : le haut fait face au poêle tandis que le bas est tourné à 450 afin de diriger les jambes vers la porte. Enfin, les enfants prennent généralement place près de la porte, faisant face au poêle, accroupis ou «à genoux ", les jambes repliées sous les fesses, et de toute façon, le dos et les pieds orientés vers le mur voisin de la porte.

On prend place sous la yourte en fonction de son statut du côté des invités ou de la famille, à droite ou à gauche, sur les côtés de l'axe vertical allant de la porte au xojmor, mais jamais sur cet axe même. Les invités sont ainsi «à la droite » des membres de la famille qui les accueille. On s'assoit du plus vieux au plus jeune, du xojmor en descendant vers la porte, les anciens prenant place dans le xojmor et les plus jeunes, près de la porte.

Outre l'endroit où il faut s'installer, les manières de s'asseoir varient également avec l'âge et le sexe. L'assise préférée des hommes, et plus rarement des femmes d'âge mûr, l'assise sur une jambe pliée avec l'autre relevée au niveau de la poitrine fait symboliquement du corps un ensemble constitué d'un siège et d'une table ${ }^{9}$. La jambe relevée ou pliée change en fonction de la place dans la yourte : la jambe levée doit être placée en direction de la porte, de manière à faire barrage aux « mauvaises influences " 
venues de l'extérieur et à s'ouvrir à celles du xojmor et de l'autel. La semelle de la jambe repliée ne doit pas être orientée vers l'autel ou le poêle, mais être placée en direction du pourtour de la yourte, des « murs » xana.

La position en tailleur est typiquement masculine. Elle est réservée aux anciens dans le contexte rituel. C'est aussi la position des divinités bouddhiques, représentées sous forme de statuettes. En revanche, l'assise dite "à genoux », le bassin reposant sur les talons, est plutôt féminine et marque le respect et la déférence.

21 Certaines assises sont propres à une activité ou à un contexte. Une technique du corps intermédiaire entre le siège et la marche (« s'asseoir en marche » javgan suu-) désigne la position accroupie. C'est une manière de s'asseoir réservée aux activités nécessitant d'être mobile au niveau du sol ou de se lever souvent. Cette assise est interdite dans le cadre d'une visite, sauf pour les enfants. Dévalorisée, elle est plutôt féminine, car largement utilisée pendant la traite; elle renvoie, en outre, aux positions de toilette et de défécation.

La yourte est un endroit où il faut de préférence s'asseoir. Néanmoins, à tout moment, on doit s'y déplacer. Généralement, on tourne autour du poêle et l'on évite autant que possible d'effectuer des trajectoires entre les "poteaux » ou "colonnes» (bagana) qui soutiennent la toiture et encadrent le poêle, que ce soit en marchant ou dans un mouvement des bras et des mains, comme lorsque l'on donne un objet. Aucun mouvement n'est donc effectué sur l'axe vertical qui conduit de la porte au xojmor, non plus que sur l'axe horizontal qui joint les deux côtés de la yourte, jamais directement audessus du poêle et entre les poteaux ou colonnes qui l'encadrent, c'est-à-dire de ce qui constitue le foyer, mais devant ou derrière lui.

\section{Les techniques de mouvement : « En plein air »}

Les deux principales techniques de déplacement, la marche et la monte, sont affectées de valeurs opposées ${ }^{10}$. La marche est peu valorisée par les Mongols, qui tirent leur fierté de leurs cavalcades équestres. Elle est associée au travail des femmes ou des enfants qui vont ramasser les bouses, le combustible animal (argal) à travers la steppe.

\section{Les démarches}

24 Les techniques de marche varient en fonction du sexe et de l'âge. L'apprentissage de la marche ne donne lieu ni à des pratiques spécifiques, ni à des exercices stéréotypés ${ }^{11}$. Il semble laissé à l'initiative personnelle, peut-être parce qu'il se déroule dans une période où l'enfant est considéré en état d'extrême fragilité12.

25 L'allure brusque et empressée n'est pas appréciée pour les hommes ${ }^{13}$. Le piétinement rapide est assimilé de manière humoristique à l'amble du cheval ${ }^{14}$. Cette comparaison fait en contrepoint valoir la démarche calme et tranquille comme la technique de marchehumaine par excellence ${ }^{15}$. Plus largement, la non-maitrise des mouvements du corps est condamnée chez l'adulte. Chez les Darxad (observations personnelles) et chez les Xalx (Njambuu et Nacagdorž, 1993, p. 8), la marche " jambes écartées » (alcgana-) ou désynchronisées, et le boitillement sont partout et tout le temps considérés péjorativement. Ce sont des signes «de vanité, de fatuité » (jorgio, sagsuu, aaligüj) ou de déficience mentale chez l'adulte. Ils sont en revanche tolérés chez le vieillard, dont la 
démarche cahotante et la perte de maîtrise des mouvements du corps sont tenues pour une annonce de la mort prochaine ${ }^{16}$. Les techniques de déplacement et, en particulier, les démarches sont donc significatives de la vitalité du corps et plus largement, de l'état de santé de la personne, de son intégrité symbolique.

Les petits pas sont plutôt une démarche de femme, prisée sur de petites distances, et les longues enjambées, une démarche d'homme, recommandée pour de longues distances ${ }^{17}$. L'opposition sexuelle des démarches recoupe ainsi une distinction en termes d'éloignement par rapport au campement et à la yourte, renvoyant doublement à la répartition traditionnelle du travail. La femme est, en effet, plutôt active au campement, tandis que l'homme assure la sociabilité élargie et extérieure à l'espace domestique.

Dans l'appréciation de la démarche d'une femme, la tenue du corps occupe une place importante ${ }^{18}$. La démarche "les bras le long du dos", signe de l'accomplissement masculin, est interdite aux femmes et aux enfants. La course est réservée aux enfants. Elle est peu estimée pour les adultes en dehors d'une activité qui la motiverait. Cela ne signifie pas que les adultes ne courent pas en cas de nécessité (par exemple pour rattraper une bête qui s'échappe), mais plutôt qu'ils ne le font guère en dehors de besoins précis. De plus, lorsqu'il s'agit d'attraper du bétail, l'éleveur est en règle générale monté et muni d'un lasso ou d'une «perche-lasso » (uurga).

Du reste, les Mongols ont une manière particulière de poser le pied à plat sur le sol lorsqu'ils courent, ce qui donne une apparence de raideur aux mouvements de leurs jambes - d'où, par exemple, cette remarque d'un Occidental de passage : « ils ne savent pas courir !» En outre, les bottes traditionnelles à bout retourné ne favorisent pas la cours $\mathrm{e}^{19}$. Les enfants, en revanche, sont valorisés pourleur maitrise de la course, tant à pied qu'à cheval. Les courses de chevaux de la fête nationale Naadam prennent, en effet, l'allure d'un rite de passage de l'enfance.

29 Ainsi les techniques de déplacement opposent les enfants aux adultes, les hommes accomplis aux femmes fécondes, les adultes en âge de se reproduire aux vieilles personnes qui ont passé cet âge.

\section{L'orientation du corps dans le déplacement}

L'orientation du corps dans le déplacement éclaire les relations entre humains ou avec des instances symboliques. Outre qu'il est considéré normal d'aller de l'avant, la marche à reculons, en dehors d'une activité la motivant spécifiquement, serait perçue comme un signe de démence chez l'adulte. De plus, les Mongols évitent de marcher en file, les uns derrière les autres: en groupe, ils préfèreront s'aligner sur une seule rangée, ou se séparer en petits groupes de personnes alignées. Dans la rangée, les cadets ont tendance à se placer légèrement en retrait, mais jamais directement derrière leur aîné.

Quand on " marche côte à côte » (zeregcen jav-), il ne faut pas marcher à la droite de la personne la plus âgée, mais à sa gauche. En principe, la partie droite du corps doit toujours être dirigée du côté de la personne la plus âgée. Que l'on soit assis ou en déplacement, la partie droite du corps représente «l'en dessus » de la personne. Le cadet respecte ainsi la droite de son aîné et se place en dessous de celui-ci, c'est-à-dire, à sa gauche (Njambuu et Nacagdorž 1993, p. 27). Ceci pose différents problèmes car, en outre, il ne faut jamais marcher sur l'ombre d'une autre personne. Le cadet doit donc faire preuve d'une grande vigilance concernant sa position par rapport à ses aînés : il 
peut être amené à changer de place à plusieurs reprises au cours d'un déplacement. Comme quand il s'agit de prendre place sous la yourte, l'aîné peut à tout moment faire comprendre au cadet qu'il a conscience de ses efforts et l'en remercie. Il autorise ainsi son "cadet " à prendre un peu de liberté par rapport aux normeslui imposant une maîtrise corporelle et une attitude de respect et de déférence dans les interactions.

Chez les Xalx, quand on marche la nuit, moment considéré comme propice pour les menées des mauvais esprits qu'il faut éviter de rencontrer, il ne faut pas regarder derrière soi, car cela peut être néfaste pour son " génie ${ }^{20}$ » (sür süld) protecteur ${ }^{21}$. C'est pourquoi tout déplacement se fait plus tranquillement de jour que de nuit.

L'orientation du corps qui se déplace est donc définie à la fois sur le plan de la latéralisation (gauche-droite) et sur celui de la perspective devant-derrière.

Enfin, il existe des attitudes privilégiées pour marquer l'arrêt. Mettre les mains aux hanches, attitude réservée aux hommes et interdite aux femmes et aux enfants, signifie le défi ou l'arrogance. C'est la posture masculine de prédilection pour l'arrêt en position debout. Pour les femmes, l'arrêt est marqué par la jonction des mains au niveau du bas-ventre, désignée par l'expression « mettre dans les manches » (xancujla-). À l'arrêt, cette position est utilisée en concurrence avec les mains pendantes le long du corps (Hamayon 1971, pp. 157-158). Ces manières de marquer l'arrêt impliquent le face à face des interlocuteurs. Il faut mettre en évidence qu'en principe, à l'arrêt les femmes mettent les mains devant le corps et les hommes sur les reins, tandis qu'ils marchent les mains dans le dos. Les techniques de déplacement semblent donc placer en opposition la perspectivité du corps des hommes et celle du corps des femmes.

\section{L'enjambement}

Les techniques d'enjambement ne semblent pas avoir retenu l'attention de M. Mauss dans l'article qu'il a consacré aux techniques du corps (Mauss 1999, pp. 365-386). Dans la culture mongole, elles sont pourtant révélatrices des représentations associées à la notion de seuil.

L'enjambement est interdit en dehors du franchissement du seuil de la yourte, de même que l'étirement est prohibé en dehors du réveil. Dans le domaine linguistique, enjamber une personne et la placer sous son pied ou sa jambe (tous deux dits xöl en mongol) signifient «l'offenser, l'opprimer ${ }^{22}$ ». D’une manière générale, quand cela est possible, on évite «de passer au-dessus » (deegüür alxa-) d'un objet ou d'une personne ${ }^{23}$. Si l'enjambement ne peut être évité, la jambe droite doit être utilisée en premier ${ }^{24}$. Ici encore, ce n'est pas l'orientation de l'objet dans l'espace ni celle du mouvement qui importe dans le déplacement, mais celle du corps par rapport à l'objet à enjamber.

Si aucune règle, sanction ou représentation populaire n'est associée aux techniques du corps relevant du sport ${ }^{25}$, de l'acrobatie ou du jeu, les positions respectives des parties du corps sur l'axe vertical importent néanmoins lors des déplacements. En ville comme à la campagne, si l'on marche sur le pied de quelqu'un, il faut lui «prendre la main ou le bras droit(e)» (baruun gar bari-) en lui touchant la «main» (gar), le « coude» (toxoj) ou l'avantbras, « l'humérus » (bugalga) pour apaiser l'offense et signifier qu'on veut « rester en paix, en amitié » (evtej java-) ${ }^{26}$ - ainsi, le conflit commencé par les pieds s'apaise-t-il toujours par une poignée de main. 
38 Marcher sur le pied renvoie à l'enjambement. Or dans l'enjambement également, le haut du corps semble constituer sa partie la plus socialisée, tandis que sa partie basse correspondrait à ce qu'il a de plus naturel. La prohibition du contact des jambes ou des pieds avec des éléments associés à la partie supérieure du corps humain (chapeau, ceinture) - comme du corps du cheval (licol, harnais, longe d'attache) - ne connaît, semble-t-il, aucune exception ${ }^{27}$. L'interdit d'enjamber vaut de manière particulière pour les femmes, compte tenu de l'écoulement du sang menstruel qui les caractérise. En somme, sous la notion de partie basse du corps, est compris l'entrejambe et, par là, le sexe ${ }^{28}$. Il est intéressant, à ce propos, de rappeler la fonction au contraire positive que peut avoir ce geste dans certaines épopées bouriates. C'est en enjambant le corps du héros laissé pour mort que sa fiancée prédestinée le "ranime », le ramène à la vie (Hamayon 1990, p. 194).

\section{Le franchissement du seuil}

Si l'enjambement d'un objet ou d'une personne peut être évité, tel n'est pas le cas du seuil de l'habitation, bosgo, littéralement " [que l'on franchit] debout ${ }^{29}$ ». Le seuil est une frontière réelle entre deux espaces, la yourte et le campement sur lequel elle est située, auxquels sont associées des techniques du corps distinctes. Franchir le seuil correspond à un changement d'attitude et d'activité, et donc de technique du corps. Le seuil est aussi une frontière symbolique entre l'espace domestique placé sous la protection des esprits ancestraux, et surtout de " [l'esprit] maitre du feu » (galyn ezen), et le monde extérieur, voué à l'errance des «esprits nuisibles " (čötgör). Aussi, le franchissement de cette frontière est-il chargé de nombreuses significations (cf. Chabros 1988 et Wasilewski 1976) et implique-t-il des techniques d'enjambement particulières.

Il ne faut ni marcher sur le seuil ni s'y arrêter ni y déposer quelque chose. L'entrée comme la sortie de l'habitation sont codifiées. Il ne faut pas buter sur le seuil, à l'entrée comme à la sortie. Si un adulte trébuche, il doit reculer et recommencer ce passage, de façon correcte. Si c'est un enfant, on prononce une conjuration qui reconnaît les bienfaits du trébuchement: c'est «un profit est entré » (olz orloo) s'il trébuche en entrant et "une perte est sortie» (garz garlaa), si c'est en sortant - de telles conjurations étaient traditionnellement prononcées quand une personne, enfant ou adulte, trébuchait, mais aujourd'hui elles sont réservées aux enfants.

Unadulte valide ne doit pas franchir le seuil à quatre pattes, comportement de mauvais augure (Njambuu et Nacagdorž 1993, p. 14). Traverser le seuil à quatre pattes n'est autorisé qu'aux très jeunes enfants ou aux vieillards impotents, deux catégories de personnes qui, ne parlant pas, se situent en dehors de la notion d'humanité. Le franchissement du seuil est donc tenu pour une marque d'humanité nécessitant un maintien du corps particulier; il n'est d'ailleurs qu'exceptionnellement autorisé aux animaux ${ }^{30}$. Pendant les funérailles, on fait marquer au corps du défunt un temps d'arrêt en passant le seuil (cf. Even 1988, Hamayon 1992, Humphrey 1999). Enfin, aucun objet ne peut être mis en travers du seuil, car il ne saurait être à la fois à l'intérieur et à l'extérieur.

42 Maintes règles définissent la conduite que doit adopter un visiteur pour que sa visite soit placée sous des auspices de bienvenue. Chez les Darxad, pénétrer dans une habitation avec la jambe gauche signifie la «diviser" (dund xagasla-), l'affaiblir, 
« l'appauvrir » (jaduura-). C'est le signe qu'on « ne reviendra pas » (daxiad irexgüj). Il faut donc ouvrir la porte avec la main droite pour rentrer du pied droit; il en est de même pour sortir. En dépit du fait que personne n'y prête attention, tous appliquent ces principes intériorisés qui connaissent assez peu d'exceptions. La latéralisation du corps double ainsi celle de l'habitation.

\section{La circumambulation} qui explique qu'elle soit moins codifiée que la marche, réservée, elle, aux espaces socialisés. C'est l'arrivée près d'un campement qui est soumise à des règles, encore remémorées par les informateurs, même si certaines d'entre elles sont aujourd'hui tombées en désuétude. Quand un visiteur arrive à cheval, on l'accueille au poteau ou à 
la "longe d'attache» (ujaa), généralement placé(e) devant la yourte, à droite en sortant $^{32}$. On lui souhaite la bienvenue en tenant sa bride, mais c'est au visiteur d'attacher lui-même son cheval. Du point de vue du visiteur qui arrive de l'extérieur, face à la yourte et en regardant vers le "nord ", il faut en principe descendre de cheval devant la yourte à gauche (ouest), côté de la yourte destiné aux invités (Njambuu et Nacagdorž 1993, p. 6). Il faut ici imaginer le cavalier face à la yourte, le devant de son corps et la tête de son cheval orientés en direction du "nord». S'arrêtant à gauche (pour lui) de la yourte, il se trouve du côté droit de la personne potentiellement assise dans le fond de celle-ci et lui faisant face, le regard tourné, quant à elle, en direction du "sud». Ainsi, la gauche du visiteur à son arrivéecorrespond-elle à la droite de l'hôte - ce qui permet de mettre en évidence le rôle du corps dans l'orientation et dans les représentations qui lui sont associées.

Du point de vue de l'hôte qui regarde vers le sud et tourne le dos au nord, l'invité arrive par sa droite, signe de respect ; et du point de vue de l'invité, faisant face au nord, l'hôte idéalement placé dans le fond de la yourte, le représentant de la famille qui l'accueille est aussi à sa droite. Par cette organisation de l'espace, les représentations de la visite placent tous les interlocuteurs dans une position de respect mutuel.

La monte est soumise aux règles sociales essentielles : droit d'aînesse et primauté masculine. Un cadet ne doit pas chevaucher devant un aîné, ni une femme devant un homme, mais rester légèrement en retrait et "en dessous ", c'est-à-dire, à sa gauche. Quand deux personnes sont sur une même route et qu'elles se séparent à un croisement, aucune des deux ne doit regarder derrière elle sous peine que " les sangles de selle ne se rejoignent plus ", c'est-à-dire, que l'une des deux ne meure. Ainsi, la face avant du corps correspondrait aux relations sociales, tandis que le dos serait plutôt impliqué dans les relations symboliques. Rappelons que les «armes» et les supports d'esprits du chamane sont cousus sur le dos de son costume rituel.

En guise de conclusion, il serait tentant de relever des associations significatives entre type d'espace, type de technique du corps et caractéristiques sociales. On pourrait associer par exemple espace extérieur non habité, déplacement à cheval, sexe masculin et absence relative de normalisation. On établirait facilement que les espaces socialisés que sont le centre de la yourte d'une part, le campement d'autre part sont les plus réglementés. On remarquerait alors que la sociabilité demande le calme et le contrôle du corps.

51 La plupart des études placent la yourte au centre des représentations de l'espace et au fondement des catégories d'orientation. À partir de sa circularité, l'environnement serait pensé comme constitué de cercles concentriques, et son orientation servirait de repère essentiel au positionnement dans l'espace (cf. entre autres, Wasilewski 1976).

C'est à partir de la position du sujet idéal - un ancien dans le fond de la yourte, ayant à sa main droite la partie de la yourte destinée à accueillir le visiteur, ouest - que l'organisation de la yourte est mise en rapport avec les points cardinaux. La désignation de la situation de la yourte dans l'espace en termes de points cardinaux se fonde sur celle de cet ego idéal, selon qu'il dirige son regard, à travers la porte, vers l'est ou le sud. Et c'est par référence à la position de ce sujet idéal que sont identifiées les places sous la yourte. Ainsi, l'orientation de ou dans la yourte correspond à celle de cet ego idéal à l'intérieur de celle-ci, son corps placé face à la porte, son dos contre celui de la yourte. 
53 En outre, plusieurs éléments indiquent que le corps et la yourte sont deux microcosmes comparables, car pensés en termes équivalents et caractérisés par les mêmes principes. La yourte est anthropomorphisée : elle possède des « pieds » (xöl), des « épaules » (mör), une "jupe» (xormoj), etc. Le corps est, comme la yourte, considéré comme une enveloppe matérielle, un "étui » censé servir de support à une âme ${ }^{33}$. Dans les conceptions des composantes de la personne des Mongols, quand un esprit prend la place d'une âme qu'il maintient prisonnière "dans un chaudron ", le corps humain squatté ne laisse sur le sol ni ombre ni empreinte. Ainsi, le corps est-il identifié par les traces de son existence concrète et de ses déplacements. Il est caractérisé par sa vitalité, tandis que la yourte l'est par sa mobilité. Comme le corps, la yourte installée pendant plusieurs semaines sur un lieu laisse des traces sur le sol. Ces traces circulaires constituent des éléments structurants du paysage : elles permettent de circonscrire le territoire de nomadisation d'un groupe. Elles offrent des points de repère précieux pour se localiser dans l'espace ${ }^{34}$.

On ne peut manquer d'être frappé par le caractère dynamique de la perception de l'espace dans la référence au corps et à la yourte. Les déplacements du corps ou du regard offrent des repères d'orientation relatifs à la position d'un ego en mouvement. L'orientation dans l'espace se fonderait ainsi davantage sur la projection de trajets et le tracé de parcours que sur la référence à des points statiques.

\section{BIBLIOGRAPHIE}

Akim, G.

1982 Mongol övörmöc xelcijn tovč tajlbar tol' [Dictionnaire abrégé des idiomes mongols] (Oulan-

Bator, Ulsyn xevlelijn gazar), $200 \mathrm{p}$.

Beffa, M.-L. et R. Hamayon

1983 Les catégories mongoles de l'espace, Études mongoles et sibériennes, 14, pp. 81-119.

Chabros, K.

1988 Space and Movement in Mongolian Culture, Journal of the Mongolian society, I, 1, pp. 30-38.

Dašdorž, Ž. et Č. Rinčensambuu

1966 Mongol cecen ügijn dalaj [Océan de proverbes mongols], (Oulan-Bator, Šinžlex uxaany

Akademijn xevlel), 140 p. [Studia folklorica, V, 5].

Gaadamba, S. et D. Cerensodnom

1978 Mongol ardyn aman zoxiolyn deež bičig [Le meilleur de la littérature orale populaire mongole]. Mongolica (Oulan-Bator), 33, pp. 31-46.

Hamayon, R.

1970 Façons de s'asseoir, Études mongoles, 1, pp. 135-141.

1971 Protocole manuel, Études mongoles et sibériennes, 2, pp. 145-207.

1990 La chasse à l'âme : esquisse d'une théorie du chamanisme sibérien (Nanterre, Mémoires de la société d'ethnologie), $880 \mathrm{p}$.

1992 Le jeu de la vie et de la mort, Diogène, 158, pp. 63-77. 
Humphrey, C.

1995 Chiefly and shamanist landscapes in Mongolia, in E. Hisch E. et M. O’Hanlon (éd.), The

Anthropology of Landscape. Perspective on place and space (Oxford, Clarendon Press), pp. 135-162.

Lacaze, $G$.

2000 Représentations et techniques du corps chez des peuples mongols. Thèse de doctorat de

l'Université de Paris X-Nanterre.

Levinson, S. C.

1996 Language and Space, Annual Review of Anthropology, 25, pp. 353-382.

Mauss, $\mathrm{M}$.

[1950] 1989 Notion de technique du corps, in C. Lévi-Strauss (éd.), Sociologie et Anthropologie (Paris, PUF), pp. 365-386 [8§ édition].

[1950] 1989 Le sujet et la personne, in C. Lévi-Strauss (éd.), Sociologie et Anthropologie (Paris, PUF), pp. 333-361 [8§ édition].

Mostaert, A.

[1941-1944] 1965 Dictionnaire ordos (New York/Londres, Johson Reprint Corporation) [2éd.].

Njambuu, X. et C. Nacagdorž

1993 Mongolčuudyn ceerlex josny xuraanguj tol' [Dictionnaire abrégé des usages prohibés des

Mongols] (Oulan-Bator, Ardyn cergijn xevlelijn « Šuvuun saaral » kompanid xevlev), 72 p.

Tangad, D.

2000 Mongol nüüdelčnij « enger zöröx » gedeg jos zanšlyn tajlbar tovčlon [Notes explicative de la coutume « disposer les rabats [des surtouts] en quinconce » des nomades mongols] (Oulan-Bator, Spb.), $21 \mathrm{p}$.

Wasilewski, J.

1976 Space in nomadic cultures, in W. Heissig (éd.), Altaica Collecta. Berichte und Vorträge der XVII. PIAC (3.-8. Juni 1974, Bonn) (Wiesbaden, Otto Harrassowitz), pp. 345-360.

Žukovskaja, N. L.

1988 Kategorii i simbolika tradicionnoj kul'tury Mongolov [Catégories et symbolique de la culture traditionnelle des Mongols] (Moscou, Nauka), 195 p.

\section{NOTES}

1. Dans son travail consacré à la «Notion de technique du corps ", M. Mauss ([1950] 1999, pp. 365-386) identifie les techniques du sommeil, celles de la veille (technique du repos: assise), celles de l'activité et du mouvement, les techniques de soin du corps, les techniques de la consommation et celles de la reproduction. Il n'en développe pas l'analyse.

2. N. L. Žukovskaja (1988, pp. 110-130) apporte des données ethnographiques sur les manières de marcher. Elle insiste sur les normes de l'étiquette associée aux déplacements et surtout à la marche.

3. R. Hamayon s'est intéressée aux «façons de s'asseoir » (1970, pp. 135-141) et au "protocole manuel » (1971, pp. 145-207). L'auteur analyse les normes régissant les « techniques de veille » et les « techniques manuelles » en termes maussiens.

4. Voir l'analyse des « catégories mongoles de l'espace » (Beffa et Hamayon 1983, pp. 81-119) et celle des « verbes de mouvement » (Chabros 1988, pp. 30-38). 
5. L'orientation absolue fait référence aux points cardinaux, ou plus largement à la position des étoiles, à la rotation du soleil, voire à la présence d'un objet culturel fixe (par exemple, La Mecque ; $c$. Levinson 1996, pp. 353-382).

6. Les Darxad sont une ethnie minoritaire vivant dans la province du Xövsgöl, au nord-ouest de la Mongolie. Ils sont connus pour avoir conservé des traditions chamaniques importantes. Ils ont aussi été «disciples» (šav), c'est-à-dire, qu'ils ont appartenu à un territoire administré par l'autorité ecclésiastique bouddhiste.

7. Dans le centre de la Mongolie, chez les Xalx, certaines femmes ne connaissent pas cet usage et utilisent la main droite pour aérer le lait. Cependant, elles se placent alors face au poêle pour orienter la coulée de lait vers le fond de la yourte.

8. L'emploi d'un oreiller est systématique. Au moment de préparer le lit d'un invité, c'est l'oreiller qu'il faut installer en premier. C'est un signe de bienvenue pour la nuit. L'oreiller joue aussi un rôle important dans les pratiques sexuelles. Celui de la femme permet de surélever le bassin pour «trotter l'amble » avec plus d'aisance (Tangad 2000, p. 21).

9. L'assise avec une jambe levée est en effet interprétée comme représentant un siège (la jambe repliée sur laquelle on s'assied) et une table (le genou de la jambe levée au niveau de la poitrine).

10. Un proverbe relativement populaire oppose ainsi ces deux techniques : «le chien fatigué pense à un os [tandis que] l'homme qui va à pied pense à un cheval » (jadarsan noxoj jas bodox, javgan xün mor' bodox; $c f$. Dašdorž et Rinčensambuu 1966, p. 115 n 11020). Mise en parallèle avec la monte, positive, la marche est négative et placée sur le même registre que la fatigue.

11. Lors de la seconde année d'âge, les Kazakhs organisent un rituel destiné à favoriser l'acquisition de la marche, ce que les Mongols ne font pas. Ces derniers, en revanche, organisent un rituel pour marquer l'acquisition de la parole.

12. En effet, l'âme de l'enfantelet n'est « pas (encore) fixée » (togtoogüj) au corps et peut donc, à tout moment, s'en échapper.

13. Un proverbe est souvent mentionné à propos des façons de marcher : "on ne devient pas sage avec des mots arrogants et l'on ne devient pas héros avec une démarche brusque " (bardam ügeer cecen boldoggüj, balmad javdlaar baatar boloxgüj ; cf. Gaadamba et Cerensodnom 1978, p. 13).

14. C'est avec les animaux domestiques, notamment avec le cheval, que se font la plupart des comparaisons de l'homme à l'animal. Les Mongols aiment à en jouer à des fins humoristiques tirant sur le sarcasme. Un « homme qui adopte l'amble » (žoroo javdaltaj xün) marche vite, à petits pas, en se trémoussant. La dépréciation de l'amble chez l'être humain s'oppose à sa relative valorisation chez le cheval, car c'est une allure qui donne stabilité et confort au cavalier. Mais la monture qui trotte l'amble est plutôt réservée aux femmes.

15. Un adage populaire dit: « le trot d'un animal sauvage n'est pas un trot et les paroles d'un alcoolique ne sont pas des paroles » (araatny xatria [sic] - xatria [sic] biš, arxičny üg - üg biš; cf.. Dašdorž et Rinčensambuu 1966, p. 9 n 6261). Les auteurs, folkloristes, restituent souvent les prononciations phonétiques de certains mots, sans mentionner le "patois local » auquel ils se réfèrent : pour xatria, il s'agit de xatiraa en xalx. Ainsi, dans le langage de l'aphorisme, le trot des animaux domestiques apparaît implicitement comme le symbole du véritable trot. Sa mise en parallèle avec la parole souligne le dressage nécessaire à son acquisition.

16. Un proverbe dit: "l'homme qui a cessé de marcher ne s'enquiert pas de son cercueil » (alxaxaa bajsan xün, avsaa surdaggüj; cf. Dašdorž et Rinčensambuu 1966, p. 7 n 6181).

17. Selon mes informateurs, les petits pas sont « dangereux » (ajuultaj) si on va loin.

18. Les mouvements du bas du corps pendant la marche possèdent, en ce qui les concerne, une connotation sexuelle alors que ceux du buste révèlent un idéal de maintien. L'expression «marcher l'amble » s'applique péjorativement à une femme qui remue excessivement le bassin en marchant et dont les trémoussements sont tournés en dérision. Et " gesticuler de haut en bas » (ocgono-) est un signe d'arrogance pour un adulte. 
19. Si les Mongols excellent dans certains sports comme le judo, la lutte, le sumo, ou le vélocyclisme, ils n'ont pas de représentant dans les compétitions de marche à pied, de course ou de saut, que ce soit en hauteur ou en longueur.

20. Sür süld « génie protecteur », combinant l'idée de talent et celle d'entité surnaturelle élective, peut prendre aussi le sens de «splendeur, majesté ».

21. "Le ciel est le protecteur de l'épaule droite et les mauvais esprits ceux de l'épaule gauche » (Njambuu et Nacagdorž 1993, p. 67).

22. L'expression "être sous le pied» (xöld baj-) signifie "être opprimé » et "s'asseoir sur des jambes " (xöld suu-) renvoie à l'idée de «se faire violence pour arriver à apaiser une situation de conflit, se faire bien voir » (Akim 1982, p. 153).

23. L'interdiction d'enjamber s'applique à tous quand il est question d'enjamber le corps de quelqu'un ou une partie de celui-ci. Il est strictement interdit de passer au-dessus des jambes d'une personne. Quand, dans l'habitation, plusieurs personnes dorment par terre, si le besoin de traverser se fait sentir, tous, du plus jeune au plus vieux, contournent les corps assoupis, quitte à devoir pour cela effectuer un tour presque complet de la yourte.

24. Certains objets suscitent des soins particuliers. Il est en général interdit d'enjamber des vêtements sous peine d'une perte de "fortune" (xišig bujan; cf. Njambuu et Nacagdorž 1993, p. 54). Cette prohibition se révèle plus forte pour certains vêtements comme la ceinture et le chapeau. En effet, bien que ceinture et couvre-chef ne soient jamais censés se trouver par terre, les enjamber ou les atteindre avec les pieds est particulièrement négatif. Chez les Mongols, l'interdit d'enjambement est exacerbé quand il concerne un objet masculin, tenu pour un attribut de virilité, par exemple la « perche-lasso » (uurga) ou le lasso de cuir (ibid., p. 47).

25. Force est de constater qu'il n'y a pas d'engouement particulier des Mongols pour des sports ou jeux de«balles du pied » (xölijn bömbög) comme le football, alors qu'ils sont férus de basketball ou de volley-ball. Dans les steppes de Mongolie, le basket-ball fait de nombreux adeptes, alors qu'en Mongolie-Intérieure ou en Sibérie, le volley-ball et le billard déchaînent des passions.

26. Le principe n'est pas de s'empoigner mutuellement la main droite et d'effectuer un balancement répétitif de haut en bas, quoi que ce soit là une tendance croissante sous l'influence russe et à l'imitation de l'usage de la poignée de main occidentale. Il s'agit plutôt d'effectuer une pression avec, au-dessus, le pouce et, en dessous, l'index et le majeur.

27. Les aliments malencontreusement enjambés sont immédiatement jetés, car ils deviennent alors impropres à la consommation. Il ne faut pas non plus mettre les pieds ou marcher sur la table sous peine de diminuer la «fortune » (xišig bujan) de la famille (ibid., p. 67). L'impureté en cause dans l'enjambement touche autant des objets porteurs de force vitale, comme les aliments, que des instruments placés sous la protection symbolique d'un esprit, comme la hache ou le poêle.

28. Il semble alors évident que les sports nécessitant un enjambement comme le saut de haies ou les courses d'obstacles ne font pas partie des techniques sportives appréciées des Mongols.

29. Le seuil est fait d'une petite latte de bois, faisant partie du châssis de la porte et permettant d'en surélever le battant. Il ouvre l'habitation au monde extérieur.

30. Les animaux d'élevage sont interdits dans la yourte, sauf les agneaux ou chevreaux nouveau-nés fragiles qui y sont mis à l'abri chaque soir de printemps pour être protégés pendant la nuit. On ne laisse jamais un animal domestique, surtout le chameau, passer la tête à travers la porte ou franchir le seuil de la yourte (Njambuu et Nacagdorž 1993, p. 46). Cela est censé attirer les mauvaises influences des « esprits » (šidel). Il ne faut pas non plus faire entrer les chiens ou les oiseaux dans la yourte car, selon une légende, une famille ayant laissé un chien et un oiseau dans sa yourte vit le feu de son foyer s'éteindre (ibid., p. 20). En ce qui concerne les animaux familiers, le chat, peu apprécié autrefois, semble occuper de nos jours une place de plus en plus importante dans les foyers mongols. 31. En Mongolie, la circumambulation est d'une manière générale associée au bouddhisme. Elle est obligatoire avant tout rituel bouddhique pour marquer la sacralité de l'espace. 
32. Il s'agit plutôt d'une longe tendue entre deux poteaux chez les éleveurs qui entraînent des chevaux de course, mais ce peut être un simple poteau, chez les autres. L'essentiel est qu'il y ait près de la yourte de quoi attacher des chevaux.

33. Les Mongols localisent l'âme dans les os et ont des représentations hiérarchisées du squelette : la tête est considérée comme le "haut du corps", tandis que les pieds en sont le «bas ». Parallèlement, sous la yourte, un axe symbolique vertical semble tracé du poêle, siège de l'esprit $\mathrm{du}$ feu domestique du foyer, vers l'anneau de compression, pièce hautement respectée censée accueillir les esprits d'ancêtres protecteurs de la famille. Un xadag, une soie d'honneur cérémonielle, y est suspendu le jour du mariage d'un couple pour consacrer rituellement leur nouvelle yourte. Celui-ci représente le « cordon ombilical » (xüj) de la yourte.

34. Pour indiquer un chemin, un Mongol fait souvent référence aux groupes présents sur le trajet : les «pâturages de X », le « campement d'hiver de $\mathrm{Y}$ », etc. Le territoire de nomadisation d'un groupe est identifié par la présence d'enclos d'hiver et de printemps, par les empreintes circulaires des yourtes sur les campements d'été et d'automne. En l'absence d'un droit de propriété clairement établi, les enclos en dur apportent une solution concrète aux conflits relatifs à l'usage des pâturages.

\section{RÉSUMÉS}

Sous la yourte mongole, l'espace est circulaire, centré sur le poêle et sans cloison interne. Il s'ouvre sur l'extérieur par une porte et une sorte de faîtière, permettant le passage de la cheminée du poêle. L'espace de la yourte comporte des lignes de démarcation implicites qui séparentles différentes catégories de lieu qui y sont identifiées. Dans cet article, l'analyse des manières de se mouvoir et de prendre place sous la yourte révèle une organisation de l'espace attribuant un lieu distinct à chaque catégorie de personne, selon son âge, son sexe et son statut social. Hors de la yourte, dans le monde environnant, des techniques du corps spécifiques servent également à marquer chaque catégorie identifiée de lieu et les frontières symboliques ou réelles les séparant. Ainsi, la référence au corps joue-t-elle un rôle important dans l'aménagement de la yourte, dans son positionnement sur le campement et, plus largement, dans l'agencement du paysage. Elle révèle le rôle du mouvement dans les perceptions de l'espace, mettant ainsi en évidence leur caractère dynamique.

The Mongol yurt forms a circular area with the fireplace in the centre. Without wall, it opens on the outside through a door and a round skylight that a chimney goes across. For all, the yurt is an area with implicit demarcation lines that divide its internal space in several places distinctively identified. On the contrary, while analysing ways of moving and acting under the yurt, this article sheds light on an area where each category of person, according to their age, gender and social status, has a specific place. Moreover, outside the yurt, in the wide world, techniques of the body are distinct one from the other according to each area and to the passage through some of the limits dividing them, either symbolic or real ones. Indeed, arrangement of the yurt, its position on the camp and likewise the perceptions of the landscape for a part refer to the body. As a result, for the Mongols, the perceptions of the environmental world seem to be dynamic and based on the notion of movement. 
INDEX

nomsmotscles Darhad, Halh, Kazakh

Index géographique : Altaï, Mongolie, Mongolie Intérieure, Sibérie méridionale

Thèmes : corps, espace, frontières, habitat, nomadisme, orientation, sédentarisation

Keywords : techniques of the body

Mots-clés : techniques du corps

\section{AUTEUR}

\section{GAËLLE LACAZE}

Gaëlle Lacaze est maître de conférences en ethnologie à l'Université Marc-Bloch de Strasbourg. Elle est rattachée au laboratoire Culture et Sociétés en Europe (Strasbourg-C.N.R.S.) et membre du CRIA (Strasbourg-C.N.R.S.) ainsi que de l'atelier Sexualité/Sexualités du LAS (C.N.R.S.E.H.E.S.S.-Collège de France) 